\title{
Proceso de aprendizaje profundo de una alumna de primaria en un contexto de alta marginación ${ }^{1}$ The Learning process of a female marginalized elementary-school student
}

\author{
Fernando Mejía Botero \\ Centro de Estudios Educativos, México \\ fernandomejia@cee.edu.mx \\ ORCID 0000-0002-5158-198X
}

\section{RESUMEN}

El objetivo del presente artículo es mostrar evidencia sobre el proceso de aprendizaje profundo de una alumna del Consejo Nacional de Fomento Educativo (Conafe) en un contexto de alta marginación y quien es acompañada por la Líder de Educación Comunitaria (LEC) mediante una relación tutora desde el modelo de Aprendizaje Basado en la Comunicación y el Diálogo (ABCD). Se usan métodos cualitativos con base en los registros de la observación "no participante" y la videograbación en aula; se exploran en profundidad las interacciones áulicas entre LEC y alumna, mediante la construcción de las secuencias didácticas para dar cuenta del sentido de los actos de habla y así derivar maneras de referirse a los objetos de aprendizaje y otros asuntos sobre los que se conversa en las aulas. El principal hallazgo de este análisis es mostrar como un hecho que la relación tutora bien implementada es una alternativa para la educación de los niños, niñas y jóvenes de las regiones más apartadas de México. Siguiendo a Mehta y Fine (2019), se muestra cómo la estudiante de este caso tiene un aprendizaje profundo, en cuanto a dominio, identidad y creatividad. Cabe advertir que este articulo no da cuenta de la representatividad de este caso en las aulas en Conafe, pero sí de que un buen aprendizaje para las poblaciones rurales es posible.

Palabras clave: aprendizaje profundo, relación tutora, Modelo ABCD de Conafe

\section{ABSTRACT}

The purpose of this paper is to share the evidence of a female student's deep-knowledge-building process in a highly marginalized school of the Nacional Council for Educational Fostering (Conafe). The student is accompanied by a Community-based Educational Leader, through a tutoring relationship, according to the pedagogical model of Learning based on Communication and Dialogue (ABCD model, due to its Spanish initials). The evidence builds on a qualitative methodology through nonparticipant observation and classroom videorecording. It studies in depth the classroom interactions between the Leader and the student through the construction of didactic sequences that allow the analysis of conversational action, references to learning objects, and other spoken issues. The main finding is the fact of a well-implemented tutoring relationship being an alternative for Mexican highly marginalized children. According to Mehta and Fine (2019), in this case, the student demonstrates a deeplearning in dominion, identity, and creativity. Research, in this case, concentrates on the possibility of adequate learning for the rural population, not on quantitative representativity.

Keywords: deep learning, tutorial relationships, Conafe's ABCD Model

\footnotetext{
${ }^{1}$ Agradezco a Dalila López Salmorán, quien hizo el levantamiento de la información del presente caso en el estudio del CEE.
} 


\section{INTRODUCCIÓN}

Este artículo presenta el caso de una alumna que lleva a cabo un proceso de aprendizaje profundo mediante la implementación del Modelo de Aprendizaje Basado en la Colaboración y el Diálogo (ABCD), que le ofrece su Líder de Educacion Comunitaria (LEC), figura educativa encargada del aula en las escuelas del Consejo Nacional de Fomento Educativo (Conafe). El texto inicia con una descripción del Modelo ABCD y de la metodología de la relación tutora que constituye su base pedagógica, para después exponer algunas consideraciones sobre la heterogeneidad de las aulas multigrado y algunos argumentos sobre el aprendizaje de niños y nińas, para terminar la parte más teórica con características del aprendizaje profundo. Posteriormente, en la parte metodológica y de datos, se describen las características de la comunidad, la escuela y el aula, así como de la LEC y de la alumna. En un siguiente momento se describe la situación áulica, mostrando cómo atiende la LEC a su alumnado y el proceso de aprendizaje de la alumna, con énfasis en la relación tutora que se lleva a cabo entre ella y su LEC. El texto finaliza con algunas consideraciones generales sobre las características del aprendizaje en esta aula.

\section{EL MODELO ABCD DE CONAFE}

El Conafe diseñó el Modelo ABCD durante 2015 y 2016, comenzó a implementarlo de manera generalizada en el ciclo 2016-2017 y funcionó hasta el ciclo 2019-2020, hasta donde se sabe. ${ }^{1}$ El Modelo $\mathrm{ABCD}$ es una propuesta que estructura las prácticas educativas de manera diferente del modelo regular. La transformación sustancial se focaliza en la relación que se desarrolla en el proceso de aprendizaje, el cual promueve la autonomía de los estudiantes.

El componente pedagógico del Modelo ABCD está enunciado en el "Marco curricular de la educación comunitaria" (Conafe,

\footnotetext{
${ }^{1}$ En Boletín No. 165 la SEP anuncia la modificación del modelo ABCD. Véase https://www. gob.mx/sep/articulos/boletin-no-165-a-traves-del-conafe-inicia-modelo-de-aprendizajecolaborativo-para-garantizar-el-logro-educativo-en-comunidades-marginadas?idiom=es
} 
2016a). ${ }^{2}$ Este documento consta de varios apartados, entre los que destacan: los propósitos, los rasgos del perfil de egreso, los principios pedagógicos, el ciclo de la relación tutora, la estructura curricular, las Unidades de Aprendizaje Autónomo (UAA) y la evaluación de los aprendizajes.

El Modelo ABCD tiene como propósitos los siguientes: brindar un estilo de formación, enseñanza y aprendizaje que asegure el derecho a una educación de calidad para los niños, niñas y jóvenes de las zonas con más altos índices de marginación; atender la diversidad presente en las aulas, a fin de que todos tengan las mismas oportunidades para aprender a aprender por cuenta propia y para toda la vida; propiciar condiciones para la comunicación y el respeto en el intercambio de opiniones y en los ritmos de aprendizaje, en la colaboración y en el interés personal por aprender; ofrecer una formación que recupere lo esencial del currículo: lectura, escritura, matemáticas, conocimiento científico y sociocultural; brindar formación intercultural con énfasis en la valoración de las lenguas y las culturas originarias; asegurar una formación que promueva la reflexión y la conciencia en sus estudiantes como ciudadanos capaces de participar activamente en sus comunidades, con responsabilidad y guiados por los valores; brindar formación a las figuras educativas con base en el diálogo y la colaboración, tal como se promueve el aprendizaje en el aula; extender la estrategia a las familias como alternativa para la superación, la formación y la conclusión de estudios truncos (Conafe, 2016a).

El modelo educativo tiene los siguientes principios: confiar en la capacidad que todos tienen para aprender y enseñar a través de la palabra; promover el encuentro cara a cara; sostener el diálogo tutor

\footnotetext{
${ }^{2}$ El Conafe está organizado operativamente en una unidad central y, hasta 2019, en delegaciones estatales. La unidad central es la encargada de diseñar el modelo pedagógico y las delegaciones de implementarlo. Para hacerlo, el Conafe organiza la formación inicial de las figuras educativas que estarán en el aula, llamadas Líderes de Educación Comunitaria (LEC); esta formación dura alrededor de seis semanas. Es importante señalar que los LEC son jóvenes que terminaron -típicamente- la preparatoria, y que después de la formación inicial prestan sus servicios en comunidades rurales con altos grados de marginación. Durante el año lectivo, las figuras LEC continúan su formación mediante reuniones mensuales o bimensuales. De igual manera, se tiene previsto operativamente que los LEC sean visitados por la figura Capacitador-tutor, que acude a las escuelas a observar cómo están trabajando y a apoyarles en el estudio de temas para que, posteriormente, puedan tutorar a sus estudiantes. La periodicidad de las reuniones de formación es decisión de las delegaciones.
} 
hasta lograr la comprensión que se busca; brindar apoyo personal adecuado a la situación particular de cada estudiante, según sus posibilidades, disposición y estilo de razonamiento; cambiar de rol -de aprendiz a tutor- para compartirlo con otros de la misma manera en que se experimentó, y ofrecer únicamente temas que se conocen con suficiente profundidad (Conafe, 2016a).

Otro aspecto del modelo ABCD es el ciclo de la relación tutora. Según López (2016), la relación tutora es un ciclo integrado por cinco momentos, en el que el punto de partida es 1) ofrecer un catálogo de temas (previamente estudiados a profundidad por el Líder para la Educación Comunitaria (LEC) o por algún alumno o alumna) para que sean seleccionados a partir del interés de estudio de cada aprendiz. 2) La tutoría se da en torno al diálogo y a las preguntas que orientan al aprendiz a hacer una exploración inicial y preconceptual del tema, a construir comprensiones, a integrar saberes y a explicitar los aprendizajes. 3) Dicho diálogo se explicita en un Registro de Proceso de Aprendizaje (RPA), en el que se escribe el proceso que se ha seguido para lograrlo y lo que se ha comprendido; esto ayuda a organizar ideas, externar dudas, anotar hallazgos, entre otros. 4) El momento de la demostración, además de fungir como evidencia de aprendizaje, permite saber si el aprendiz está en condiciones para ser tutor de ese tema en concreto. 5) Los temas que el alumnado aprende se constituyen en su "catálogo de temas", sobre el que está en capacidad de ofrecer y brindar tutoría a otra persona.

El RPA y la demostración pública son elementos fundamentales en la evaluación, ya que en ellos se describen los procedimientos seguidos, lo que se aprende y cómo se aprende (ejercicio de metacognición), lo cual constituye una evidencia de aprendizaje. Es posible evaluar el aprendizaje de todo el tema mediante la revisión del RPA y a través de su exposición oral con la demostración pública. Por su parte, el cambio de rol genera una polivalencia en el aprendizaje. De acuerdo con Rincón Gallardo (2012), lo que se gana en la rotación del rol es que compartir el tema permite transparentar el proceso de aprendizaje, descubrir las propias dificultades y, con una guía pertinente, desarrollar nuevos aprendizajes y ganar confianza en sí mismo. Para López (2016), la tutoría entre colegas ayuda en el proceso de comprensión del mismo tema, y tiene doble propósito. 
En principio, crea una situación que permite al tutor fortalecer sus estrategias de comprensión, al enfrentarle a un esquema de pensamiento distinto del suyo, con lo que pone a prueba qué tanto de su experiencia de aprendizaje le permite ayudar a otro a aprender un tema. El segundo propósito es organizativo ya que, como la relación tutora implica atención personalizada, sería imposible que el docente pudiera brindarla si fuera el único tutor. Así, todos aprenden de todos y, por tanto, los roles para la enseńanza y el aprendizaje entre tutor y aprendiz se reconfiguran creando una comunidad que se dispone a aprender y a compartir.

Por último, el modelo $\mathrm{ABCD}$ también tiene las Unidades de Aprendizaje Autónomo (UAA), que son los propios materiales didácticos, que se tiene previsto que estén en las aulas como apoyo didáctico. Están organizadas en cinco campos formativos: Exploración y comprensión del mundo natural, Exploración y comprensión del mundo social, Lenguaje y comunicación, Pensamiento matemático y Participación en comunidad (Conafe, 2016c). La estructura de las UAA presenta un menú temático donde están explícitos el campo formativo, la dimensión curricular, los temas, las unidades de aprendizaje y los ejes transversales; y es precisamente de este menú donde entre LEC y estudiantes eligen los temas que desean estudiar. A su vez, cada tema tiene una presentación (las más de las veces, a manera de pregunta), el propósito general y los específicos, diferentes desafíos a atender por parte del aprendiz, y recordatorios para registrar el proceso (Conafe, 2016c).

Según Mejía, Pérez y Frausto (2016) la relación tutora, como base del Modelo $\mathrm{ABCD}$, posee las siguientes características:

Para quien aprende

- El aprendizaje se hace a partir del interés de quien aprende, pues es quien escoge el tema que desea trabajar.

- Tiene nociones sobre lo que va a aprender, y las pone en juego para pasar a su zona próxima de aprendizaje.

- En el proceso de aprendizaje se activan las facultades y conocimientos de quien aprende a partir de un desafío.

- Lo hace a su ritmo. 
- Pone en juego sus facultades de indagación, búsqueda de información y lectura dada la necesidad de hacer acopio de información para resolver el reto.

- Desarrolla la escritura de manera práctica gracias a la condición de hacer el registro del proceso de aprendizaje.

- Desarrolla la habilidad de aprender por cuenta propia.

- No cesa de trabajar en el tema hasta que puede hacer la demostración pública.

- Una vez demostrado el aprendizaje, puede asumir el rol de tutor y seguir aprendiendo sobre el tema.

\section{Para quien tutora}

- Sabe lo que enseña y además lo enseña tal como lo aprendió (mediante la tutoría).

- Identifica en quien aprende las nociones o saberes previos sobre el tema que escogió como reto.

- Trabaja mediante preguntas, retos, analogías, ejemplos que permiten que, quien aprende, logre el vínculo entre lo que sabe y lo que está aprendiendo.

- Interviene para saber qué piensa el/la aprendiz, y en ocasiones (las menos), para indicar un camino a seguir para que resuelva el reto.

- Establece una relación afectiva con el/la aprendiz en un plano de igualdad, respeta sus ritmos y permite que exprese abiertamente dudas, dificultades, retrocesos y avances.

- Acompaña a quien aprende hasta que logra satisfacer su aprendizaje.

Lo anterior permite afirmar que las prácticas educativas que se desarrollan dentro de las redes de tutoría (RT) requieren la interacción horizontal entre tutor y aprendiz; en dicha relación no jerárquica se explicitan los procedimientos, pues se trata de que cada aprendiz pueda apropiárselos como una herramienta que utilizará al convertirse en tutor.

De acuerdo con Cámara, la pedagogía de las RT puede entenderse como: 
Un contrato personal entre quien posee una competencia concreta y quien desea adquirirla. El contrato se caracteriza por la decisión de dos personas de dedicar el tiempo disponible al propósito central de adquirir un conocimiento y practicar una competencia. En una relación tutora no hay interferencia de actividades o propósitos distintos de los establecidos en el convenio. Para el aprendiz, el empeño se justifica por sí mismo, al menos mientras se ejercita, independientemente del uso que pueda dar después a lo que aprende. La acción misma es parte evidente del logro y fruición de lo que vendrá después de manera más plena y satisfactoria (Cámara, 2013, s. p.).

Una característica fundamental es que la relación personalizada en las RT permite construir el "aprendizaje desde el error", lo cual supone usar los circuitos de aprendizaje que sigue el estudiante para afianzar lo que está aprendiendo. El error es un elemento importante, porque quien aprende tiene la oportunidad de analizar la secuencia lógica que usó para construir una afirmación y no puede continuar hasta que escudriñe y comprenda comparativamente la lógica que lo llevó a un error y a la lógica que implica un acierto; quien tutora debe cerciorarse de que el aprendiz identifique los elementos específicos que lo llevaron a ese desarrollo y reconozca si ha sido una elaboración correcta: de lo contrario, que replantee una nueva manera de resolverlo para lograr el objetivo de estudio.

Todas las escuelas Conafe son multigrado, es decir, aquéllas donde está presente alumnado de diferentes grados escolares. Según la cantidad de docentes, la escuela puede ser unitaria, bidocente, tridocente, tetradocente o pentadocente. Esta definición se ocupa de una condición y no resalta la característica primordial de estas aulas: la heterogeneidad de los estudiantes.

La atención a la heterogeneidad en el alumnado implica que los docentes inicien sus intervenciones pedagógicas desde el lugar donde se encuentran sus alumnos y no desde la pretensión curricular de un mismo texto o clase para todos; de igual manera, asumen la condición ineludible de que las y los estudiantes son muy diferentes entre ellos en aspectos trascendentes para el aprendizaje, como son sus aptitudes, sus intereses y su perfil (Tomlinson, 2008). 
Para lograr esto deben proporcionar oportunidades de aprendizaje que permitan su involucramiento activo y aprender a su ritmo (y no al impuesto y normalizado para todos en el aula). Estas características de las oportunidades de aprendizaje son dos de las referidas por Vosniadou (2006): Participación activa. El aprendizaje requiere la participación activa y constructiva del estudiante. Dar tiempo para la práctica. Aprender es una tarea cognitiva compleja que no puede ser apresurada, requiere un tiempo considerable y periodos de práctica para comenzar a construir experiencia en el área, y Diferencias de desarrollo e individuales. Los niños aprenden mejor cuando sus diferencias individuales son tomadas en cuenta. Otras de las características que esta autora señala acerca de Cómo aprenden los niños [y las niñas] se describen a continuación.

Actividades significativas: las personas aprenden mejor cuando participan en actividades que perciben como útiles en la vida cotidiana y que culturalmente son relevantes.

Relacionar nueva información con conocimiento previo: el nuevo conocimiento es construido sobre las bases de lo que ya se entiende y se cree.

Uso de estrategias: el empleo de estrategias efectivas y flexibles ayuda al estudiante a entender, razonar, memorizar y resolver problemas.

Autorregulación y reflexión: los estudiantes deben saber cómo planear y monitorear su aprendizaje, determinar sus propias metas de aprendizaje y corregir sus errores.

Reestructurar el conocimiento previo: los estudiantes deben aprender cómo resolver las inconsistencias internas y, cuando es necesario, reestructurar los conceptos preexistentes, entre otras.

Atender de manera adecuada las anteriores características de las aulas rompe con la "gramática escolar" prevista por el sistema educativo, el cual organiza el currículo, los materiales, la formación docente -inicial y continua-, la enseñanza, la evaluación y la certificación bajo la premisa de la supuesta "homogeneidad" que da grupos de alumnos de la misma edad (Tyack y Cuban, 2002). Así, los y las estudiantes son tratados como un "grupo homogéneo" capaz de seguir, al unísono, un plan de estudios estrictamente prescrito para el año académico y establecido en las guías para maestros y alumnos, donde los libros de texto gratuito (LTG) y del maestro marcan el 
ritmo, el contenido y los métodos de enseñanza y aprendizaje para cada lección, lo que brinda pocas oportunidades de diferenciación entre los alumnos (urbanos y rurales, con mayor y menor rendimiento).

Atender la heterogeneidad real en las aulas poniendo el aprendizaje en el centro implica un giro hacia el aprendizaje profundo, lo cual implica un movimiento cognitivo y uno de dominio; el cognitivo va de los conocimientos previos al conocimiento nuevo (aprendizaje) mediante un doble tránsito: la profundización (establecimiento de relaciones de dicho conocimiento con otros más específicos de la misma disciplina) y la extensión (vinculación de este saber disciplinario con otras disciplinas y con la vida diaria del estudiante, que se conoce como "aprendizaje significativo"). Por su parte, el movimiento de dominio se da cuando las operaciones mentales que se ejecutan son del orden de "dar explicaciones, mostrar evidencias y ejemplos, generalizar, aplicar a situaciones nuevas, establecer analogías, representar ese conocimiento en forma diferente, usarlo para resolver problemas de la vida cotidiana, avanzar en el conocimiento estableciendo relaciones inusuales" (Valenzuela, 2008, p. 1).

\section{APRENDIZAJE PROFUNDO}

El aprendizaje profundo es una estrategia pedagógica que contribuye a que el estudiantado aprenda a aprender, mediante la promoción de la indagación, la investigación, el diálogo y la participación del o la estudiante. Se contrapone al aprendizaje superficial, que es más mecánico, pasivo y unidireccional, donde quien enseña es quien da las indicaciones pautadas de las actividades a realizar, o incluso las explicaciones de los procedimientos a realizar.

En el trabajo de Soler, Cárdenas, Hernández y Monroy (2017) se hace una descripción del origen y evolución de los enfoques de enseñanza y de los de aprendizaje; ellos plantean un paralelismo entre el aprendizaje profundo y el aprendizaje superficial. Es importante seńalar que ambos son enfoques del aprendizaje, es decir, "se refieren a los modos en los que el alumnado afronta el proceso de aprendizaje en un contexto determinado" (Sigüenza, Arsuaga, García y Martínez, 2015, p. 928). Estas mismas autoras plantean una diferenciación entre enfoque profundo: "motivación intrínseca, 
pensamiento crítico, búsqueda de conexiones entre conocimientos, comprensión de conceptos, etc.", y un enfoque superficial de aprendizaje: "memorización, motivación extrínseca, estudiar para aprobar, reproducción de contenidos, etc." (Sigüenza et al., p. 930).

Para Valenzuela (2008, pp. 1-2), aprender de manera profunda implica, "por una parte, el establecimiento de relaciones significativas entre los conocimientos previos y la información que debe llegar a constituirse en conocimiento, a través de las dinámicas de profundización y de extensión”. La profundización es el establecimiento de relaciones con elementos más específicos de la misma disciplina y la extensión con relaciones con elementos de otras disciplinas y con la cotidianidad o contexto del estudiante. También implica el dominio y, citando a Beas, Santa Cruz, Thomsen y Utreras (2001), plantea que es la ejecución de otras operaciones mentales de orden superior "tales como dar explicaciones, mostrar evidencias y ejemplos, generalizar, aplicar a situaciones nuevas, establecer analogías, representar ese conocimiento en forma diferente, usarlo para resolver problemas de la vida cotidiana, avanzar en el conocimiento estableciendo relaciones inusuales".

La profundización, la extensión y el dominio que lleve a cabo un estudiante dependerá de las situaciones de aprendizaje a que se enfrente, y en general estas situaciones están pautadas por las de enseñanza; en otras palabras, quien aprende se enfrenta a retos cognitivos en ambientes motivantes, $\mathrm{y}$ ambos dependen de manera importante de quien enseńa. Biggs (1993) afirma que el aprendizaje resulta de la interrelación de tres elementos clave: la intención (motivo) de quien aprende, el proceso que utiliza (estrategia) y los logros que obtiene (rendimiento). Por tanto, quien enseña y quien aprende son corresponsables del proceso y del resultado del aprendizaje: quien enseńa dando la estructura de las condiciones de aprendizaje, y quien aprende implicándose en ellas; con ello desentraña el núcleo de las relaciones de aprendizaje: quien aprende, quien enseńa y la tarea. Con lo anterior, Biggs (1993) plantea tres enfoques: a) enfoque profundo o comprensión real de lo que se aprende; b) enfoque superficial o reproducción de lo que se enseña para atender los requisitos mínimos, y c) enfoque de alto rendimiento o logro, cuyo objetivo es maximizar las calificaciones. Sin embargo, según López 
y López (2013, p. 136) "en la última década, las investigaciones llevadas a cabo por diversos autores, incluido Bigss, han modificado este modelo trifactorial en un modelo de dos enfoques de aprendizaje: uno orientado a la comprensión y el significado, y otro hacia la superficialidad y reproducción, considerando que el enfoque de logro no posee suficiente entidad para destacarse como un enfoque diferenciado y que podría integrarse en uno de los dos enfoques".

En un trabajo de Mehta y Fine (2019) titulado In Search of Deeper Learning: The Quest to Remake the American High School, se presenta la evidencia de visitas a escuelas en Estados Unidos y se muestra que, en general, esas escuelas no tenían nada de extraordinario, estaban atrapadas en la "gramática escolar", pero al mismo tiempo encontraron casos aislados de prácticas pedagógicas que lograban involucrar a los y las estudiantes en situaciones cognitivamente complejas. También encontraron que gran parte de esas experiencias ocurrían no en las clases del currículo base, sino en las de periferia: optativas, clubes, extracurriculares, y que ello les permitía tener una práctica fuera de la gramática escolar. Uno de los puntos relevantes para el presente artículo es que llegaron a la conclusión de que una

visión distinta del aprendizaje profundo, no sólo en la escuela, sino en la vida, surge en la intersección de tres virtudes: dominio, identidad y creatividad. En los espacios que los maestros, los estudiantes y nuestras propias observaciones identificaron como los más convincentes, los estudiantes tuvieron oportunidades de desarrollar conocimientos y habilidades (dominio), llegaron a ver su yo central como vitalmente conectado con lo que estaban aprendiendo y haciendo (identidad), y tuvieron oportunidades de poner en práctica su aprendizaje produciendo algo (creatividad) en lugar de simplemente recibir conocimiento. A menudo estos espacios o aulas se regían por una lógica de aprendizaje; los estudiantes tuvieron la oportunidad de hacer cosas (periódicos, colecciones de poesía, documentales, producciones teatrales, presentaciones de debate) bajo la supervisión de profesores y/o estudiantes mayores que modelarían los pasos creativos involucrados, proporcionarían ejemplos de trabajo de alta calidad y ofrecerían retroalimentación precisa Mehta y Fine (2019, s. p.). 
De lo anterior se resaltan los tres elementos del aprendizaje profundo: dominio, identidad y creatividad, pues serán éstos los que soportan el análisis posterior de la práctica analizada.

\section{METODOLOGÍA}

El presente texto es un derivado de un trabajo más amplio del Centro de Estudios Educativos acerca de las prácticas pedagógicas en aulas de escuelas comunitarias, que a su vez está inmerso en la Evaluación de las habilidades metacognitivas de LEC de cero y un año de experiencia y alumnos de $5^{\circ}$ y $6^{\circ}$ de primaria. $^{3}$

El análisis de este caso sigue métodos cualitativos con base en los registros de la observación "no participante" y la videograbación en las aulas, y aquí se exploran en profundidad las interacciones áulicas entre LEC y alumna. ${ }^{4} \mathrm{El}$ caso fue elegido por ser uno en el que se observó la mejor expresión de la relación tutora de entre los observados, tanto en lo que respecta al caso concreto con la estudiante determinada, como por la manera en que se desempeñaba en el aula en término generales. Se trata de un caso donde se expresaron varios de los elementos teóricos planteados: aula altamente diversa, atención a la heterogeneidad, desempeño de la LEC y "visibilidad" del aprendizaje profundo con las tres características planteadas por Mehta y Fine (2019): dominio, identidad y creatividad.

La recolección de información se realizó mediante los siguientes instrumentos: guía de observación del contexto de la escuela, registro de videograbación de interacciones áulicas durante jornada escolar y media, registro fotográfico del aula y el RPA, entrevistas a alumnos, LEC y Capacitador tutor. ${ }^{5}$

\footnotetext{
${ }^{3}$ Esta evaluación fue financiada por el Conafe. Se llevó a cabo durante el ciclo escolar 2019-2020. Consistió en el análisis al inicio y al final de ese ciclo de habilidades metacognitivas en muestras representativas a nivel nacional de LEC y alumnos del Conafe. La parte cualitativa del estudio fue financiada por el CEE, y se trató de la observación de prácticas pedagógicas de igual número de LEC, seis escogidas con base en los resultados polarizados de alumnado y las otras seis con base en los resultados de LEC, también polarizados.

${ }^{4}$ En la parte de la descripción usaremos el nombre ficticio de "Karime" para la alumna, con el objetivo de facilitar la lectura y al mismo tiempo mantener la confidencialidad de los datos.

${ }^{5} \mathrm{El}$ acompañamiento de esta figura educativa durante la visita tenía como propósito permitir que observara los mismos eventos que la investigadora, con el fin de indagar qué había visto, qué le parecía y cómo retroalimentaría a la LEC.
} 
La visita a la escuela fue de dos días, en los cuales se videograbó la relación tutora durante jornada y media, y en la otra media jornada se registró la demostración pública de la alumna objeto central del presente texto; además, se realizaron entrevistas.

Para el análisis de la práctica pedagógica se usaron varios referentes metodológicos. La construcción de las secuencias didácticas desarrolladas durante la observación y videograbación se define como la descripción de lo observado y escuchado en el tiempo registrado e incluye: interacciones lingüísticas, tema trabajado, descripción de las actividades realizadas, tiempo de las actividades, entre otras (Mejía y Sierra, 2008). Con este tipo de análisis se da cuenta del sentido de los actos de habla y de ello derivar maneras de referirse a los objetos de aprendizaje y a otros asuntos sobre los que se conversa en las aulas. El proceso es construir segmentos de habla con sentido propio y secuenciar diacrónicamente lo que sucede en las aulas. También permite obtener información acerca de aspectos como la forma de retroalimentación, de andamiaje, de actividades de análisis e investigación. En general, se trata de reconstruir cómo son los diálogos instructivos, si éstos se dan centrados en el contenido o si se encadenan ideas para lograr una comprensión más profunda del mismo (planteamiento de preguntas, integración de los saberes previos con los nuevos, reconocimiento y reiteración de ideas clave). Se tiene en cuenta de manera particular si las y los estudiantes tienen un papel activo en estos diálogos.

\section{La aprendiz y contexto}

A continuación se presenta una descripción de "Karime", del aula y la escuela a la que asiste, así como de la LEC que la atiende.

\section{Acerca de Karime}

La alumna es una estudiante de $6^{\circ}$ de primaria, ha estudiado toda su primaria en Conafe. Manifiesta querer seguir estudiando y llegar hasta posgrado. Menciona tener un frecuente apoyo para tareas de sus familiares, su padre tiene primaria incompleta mientras que su madre, primaria completa. Habita en la comunidad donde se ubica 
la escuela, la localidad es rural, muy pequeña y con alto índice de marginación.

Respecto a la experiencia que la alumna ha tenido con el ciclo del modelo $\mathrm{ABCD}$, ella manifiesta que la mayoría de las veces escoge los temas que quiere estudiar por sí misma, que ha recibido todas las tutorías de la LEC, que habitualmente ha elaborado el RPA y que en la mayoría de los temas estudiados ha hecho demostraciones públicas; desde hace poco ${ }^{6}$ empezó a dar tutorías a compañeros de grados inferiores al suyo. Lo anterior es indicativo de que con esta alumna el ciclo de tutoría que plantea el modelo se cumple y sólo quedaría pendiente que recibiera tutoría de algún compañero.

\section{La líder de educación comunitaria}

La LEC manifiesta tener 30 años; terminó la preparatoria, está estudiando una licenciatura en ciencias sociales y administrativas y espera cursar un posgrado. No es originaria de la comunidad donde está ubicada la escuela y tampoco es hablante de lengua indígena (HLI).

La LEC informa que éste es su primer ciclo de trabajo en Conafe. Ella sola atiende a alumnado de preescolar y primaria en una misma aula. Comenta que conformó el catálogo de temas al inicio del ciclo con las cuatro UAA que estudió en las seis semanas de formación inicial, previas al ciclo escolar. Al momento de la visita, dice tener listas para ofrecer a sus alumnos entre cuatro y seis UAA de cada uno de los cuatro campos formativos, las que estudió en las reuniones de tutoría. Respecto a esas reuniones, manifiesta que durante el ciclo escolar 2018-2019 estuvo en reuniones de tutoría menos de cinco días y que el tiempo en ellas se dedicaba principalmente al estudio de las UAA. De igual forma, declaró que fue visitada por el capacitadortutor tres veces y que cada visita duró en promedio cuatro días.

Respecto al ciclo de la relación tutora en el aula, señala que en ocasiones las alumnas y alumnos escogen los temas que se van a estudiar, y algunas veces los escoge ella. La mayor parte del tiempo ella es quien da la tutoría al alumnado, pero ya hay tutoría entre alumnos.

\footnotetext{
${ }^{6}$ El levantamiento fue llevado a cabo durante el mes de abril de 2019.
} 


\section{El aula y la escuela}

El aula tiene una serie de materiales didácticos colocados en las paredes con el propósito de generar un ambiente alfabetizador: el abecedario, el reglamento del aula, el autopase de lista, los colores, las estaciones del año, los números en unidades, decenas y centenas, los animales terrestres y acuáticos, una biblioteca de aula y el "palabrario". Respecto del Modelo ABCD están los campos formativos, los temas ofertados, así como la red de tutoría que están conformando. El aula también cuenta con una computadora y un pizarrón. Las y los estudiantes usan mesas rectangulares de fácil movilidad. Hay suficientes UAA para apoyar el estudio de todas y todos.

Esta escuela Conafe atiende a dos comunidades, una donde se ubica la escuela y otra de una comunidad indígena ubicada a 45 minutos, donde antes tenían servicio del Consejo, pero se cerró por disminución de la matrícula. Lo anterior hace que la mitad de la población escolar sea HLI. La LEC menciona que el alumnado sí usa lengua indígena en el aula.

\section{RESULTADOS}

A continuación se presentan los resultados del análisis de los registros: 1) se describen algunos aspectos de lo sucedido en el aula y 2) se describe el proceso de aprendizaje de la alumna con el objeto de evidenciar cómo es atendida un aula multigrado y diversa, al tiempo que se atiende en relación tutora a una alumna.

\section{Un aula en redes de tutoría}

La LEC está ubicada al centro del aula; los alumnos están distribuidos por el resto del salón, unos están sentados solos y otros en parejas a sus mesas. Esto permite que la LEC esté, de alguna manera, equidistante de todos sus alumnos. Unas veces los alumnos van a donde ella está, otras veces es la LEC quien se desplaza a observar y atender a sus alumnos.

Así pues, mientras la LEC da tutoría a Karime, un alumno de $6^{\circ}$ da tutoría a una alumna de $2^{\circ}$ y el resto del alumnado trabaja por cuenta propia; el tiempo y la atención de la LEC se distribuye en 
estos tres focos. Por ejemplo, en varias ocasiones la LEC se interesa en cómo va la tutoría entre alumnos; en una ocasión, el tutor le muestra el producto de la alumna, el cual consiste en poner las figuras geométricas encima del respectivo nombre, la LEC le pide que lo desarme, porque ella quiere verificar cómo lo realiza la alumna de $2^{\circ}$ grado. De esta manera, la LEC constata que esta alumna ya sabe los nombres correctos de dichas figuras. Esta descripción es una muestra de que la LEC sabe que las tutorías entre alumnos deben ser observadas y apoyadas por ella.

En otro momento, un alumno de $3^{\circ}$ grado hace una indagación acerca de la fauna mexicana, en particular el gato montés; la LEC le pide que escriba dónde vive y de qué se alimenta el felino. El alumno escribe en su libreta "vive en la selva y se alimenta de hojas y otros frutos del bosque". La LEC le indica que busque en computadora dónde vive y de qué se alimenta el gato montés, que lo escriba en su libreta y compare con lo que ha escrito antes; el alumno busca en la computadora y, cuando termina, le dice a la maestra que el gato montés vive en la selva y que es un animal carnívoro. Esta intervención de la LEC indica cómo la maestra aprovecha el "error" del alumno para promover no sólo la indagación de información, sino la verificación por él mismo de la veracidad de sus preconceptos, permitiendo una construcción precisa del conocimiento; la LEC "no corrige", sino que da un andamiaje al alumno.

También cabe mencionar que varias alumnas y alumnos se acercan a la LEC para mostrarle sus productos, hacerle preguntas, y ella responde, en general, en la misma dirección pedagógica de las dos descripciones anteriores.

\section{Relación tutora a Karime}

A continuación se hace una descripción de la relación tutora. El tema escogido por Karime fue "Biodiversidad" (Conafe, 2016b). Lleva dos semanas y media estudiándolo. Durante este tiempo, tal como fue registrando en el RPA, trabajó los siguientes contenidos: "Tipos de animales", "Biodiversidad", "Biodiversidad en mi localidad", "Ecosistema”, "Genes", "Ecosistema de México”. Durante la observación, trabajó los contenidos "Especies endémicas", "Cómo es el ecosistema de mi comunidad", "Ecosistema natural y artificial". 
Karime registra: "decido elegir esta unidad, ya que me parece muy interesante porque la diversidad de especies en nuestro planeta vale la pena tomarnos el tiempo para dedicarles a su estudio y analizar a la vez el motivo del porqué algunas especies empiezan a extinguirse. A la vez quizá nos pueda ayudar a buscar opciones para apoyar o ayudar a cuidar estos animales". Allí mismo explicita su propósito de aprendizaje: "identificar plenamente la biodiversidad en sentido general y especialmente en nuestro país. Aportar a lo que se pueda para el cuidado de los mismos".

Después, Karime sale del aula a observar su entorno; de esta experiencia escribe lo que se muestra en la imagen 1.

- Imagen 1. Observación de Karime a su entorno

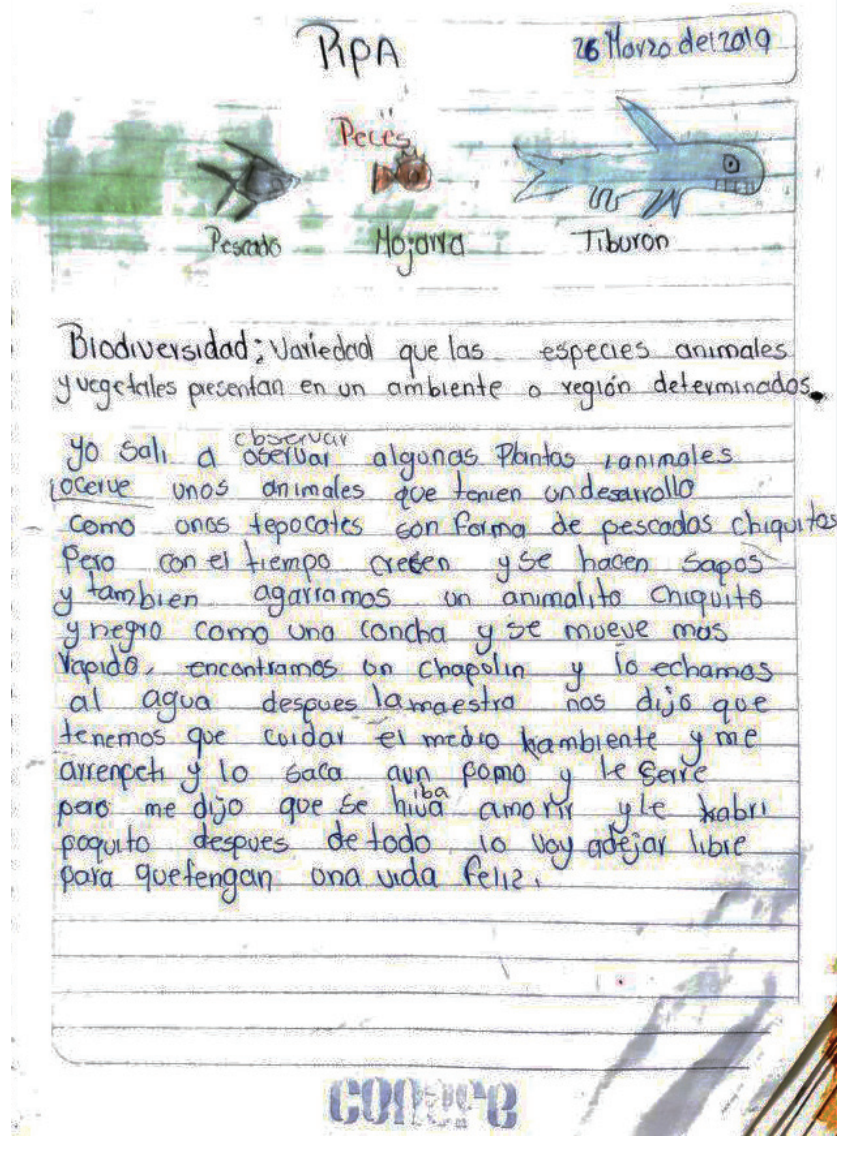


La LEC y Karime revisan en la UAA cuántas especies hay en México, la LEC le solicita que lea la información y haga una gráfica de los datos que vienen en la unidad; Karime construye una gráfica de barras con la cantidad de especies existentes en México, la maestra revisa la gráfica y le pregunta “¿de cuál hay más, entonces?” Karime responde "de invertebrados", la maestra asiente. Posteriormente le indica que haga su registro "de qué fue lo que aprendió, de qué se dio cuenta cuando graficó, de cuáles había más”, Karime procede a escribir en su libreta. Como proceso de aprendizaje, la LEC promueve en la alumna la transformación de la información en gráfica de barras, y también se ocupa de que la alumna explicite por escrito lo que aprendió, haciéndolo "visible".

Luego se presenta la siguiente secuencia:

LEC: $\quad$ Hay que empezar a leer lo de las especies endémicas, ¿sabe qué es endémico?

Karime: No.

LEC: ¿Qué cree que es una especie endémica?

Karime: Lee en la UAA: "Otro de los rasgos importantes de la riqueza biológica de México es que muchas de sus especies son endémicas. Esto quiere decir que su distribución está restringida a una determinada región o país y no se encuentran en algún otro lugar del planeta. México no sólo tiene especies endémicas, también existen géneros o familias endémicas." (Mientras la alumna lee, la LEC va donde está el alumno-tutor y la alumna-aprendiz).

LEC: ¿ ¿Ya supo cuáles son las endémicas? Si no, hay que agarrar un diccionario.

Karime: Son los animales que casi no hay.

LEC: Ok. Entonces escriba: investigación, especie endémica y pone lo que usted considere que es.

Karime: (escribe en su libreta). Maestra ya.

LEC: ¿Ya?

Karime: $\quad$ Es que en el país, nomás en el país hay de eso.

LEC: Ajá (la LEC se levanta a atender a un alumno).

Karime: (la alumna vuelve a leer el texto de la UAA; luego se 
levanta por un diccionario). Maestra no viene en el diccionario.

Como se aprecia, la LEC incentiva que la alumna se acerque a los conceptos mediante la lectura pausada, que explore fuentes de información que tiene a la mano, y luego promueve que escriba un texto sobre su propio entendimiento; en su RPA escribe: "son animales que sólo en algunos países hay, no en todos lados y no hay muchos...". Al comparar lo que viene en la UAA ("que su distribución está restringida a una determinada región o país y no se encuentran en algún otro lugar del planeta") y lo escrito por ella, se aprecia que hay una transformación del lenguaje, mantiene la esencia de la definición.

LEC: Ni viene verdad, nos vamos a quedar con lo que usted me dijo, porque sí es eso, son especies...

Karime: Que no hay muchas.

LEC: Que no hay muchas y pertenecen a determinado lugar. Y a veces como esas especies son muy bonitas, los humanos tratamos de agarrarlas o comérnosla y como casi no hay, son las que están...

Karime: En peligro de extinción.

De igual manera, la LEC da cierto andamiaje a la alumna al precisarle que "pertenecen a determinado lugar", y expande el contenido al relacionarlo con el factor antropogénico de pérdida de biodiversidad.

LEC: Vamos a dejar así, ya con lo que tienes está bien. Ahora vamos a checar las especies endémicas que hay en México.

Karime: O hago una gráfica de esto (señala la información de la UAA donde se especifican los porcentajes de especies endémicas y no endémicas en México de anfibios, reptiles, mamíferos, aves, peces y plantas vasculares).

LEC: $\quad$ Primero hay que leerlo, ¿¿no? Primero le damos una leída, ahí donde dice "especies endémicas", ahí es donde inicia. 
Karime: ¿Usted lee o yo?

LEC: $\quad$ Empiézale.

La LEC acompaña a Karime en el proceso de manera pausada, pues aunque ella quería hacer una gráfica con la información de la UAA, la LEC considera prudente leer todo el texto sobre las especies endémicas para tener más información.

En el RPA la alumna da cuenta de que sigue la pauta que marca la UAA: lee el texto de la UAA donde viene la información del contenido; extrae de ella lo que le permite construir su conocimiento respecto al tema; registra con sus propias palabras la comprensión de lo leído; realiza las actividades que le propone la UAA y, por último, registra qué aprendió y qué se le dificultó. Cuando tiene alguna duda acude a la LEC para solventarla; la LEC, en general, le responde con alguna pregunta o con la indicación de que encuentra la información en la UAA o en otro material didáctico (diccionario, computadora); es decir, en general, la LEC no da respuesta a sus inquietudes, procurando con ello que la alumna continúe la indagación.

Una manera de apreciar con detalle el proceso es siguiendo lo que Karime escribe en su libreta como RPA. Así, en varios contenidos se aprecia que no copia la información de la UAA; por ejemplo, registra en su libreta (indicado en la UAA) algunas "especies endémicas de México" y "cómo harías para cuidarlas y protegerlas", Karime escribe: el cocodrilo, la tortuga de mar, la ballena, la guacamaya, el tiburón, las algas y, sobre ellas, cómo protegerlas, por ejemplo, acerca de las algas escribió: "son plantas muy bellas que de ellas solamente se encuentra en profundas, se puede decir que ellas también reproducen bellos peces de colores y si las cortan no va haber pescados de muchos colores". Enseguida, escribe sobre lo que aprendió respecto a estos contenidos: "Yo aprendí de toda la lectura que hay muchos animales en vía de extinción y tenemos que cuidarlos, también cuáles son las especies que hay más en nuestro país y que no en todos los países se pueden encontrar, es por eso que los tenemos que cuidar, porque en nuestro país hemos obtenido el tercer y el cuarto en algunos animales en peligro de extinción. También aprendí todos los ecosistemas que podemos encontrar. También es la palabra genes, es el tipo de $\mathrm{ADN}$ que tienen las personas y los animales no 
todos tienen el mismo". Después registra sus dificultades: "se me dificultó qué era el $\mathrm{ADN}$, pero lo resolví investigando con la maestra y también nos juntamos todos mis compañeros y lo resolvimos". Al explicitar sus dificultades, construye la capacidad de regulación sobre su aprendizaje. Por otro lado, encontrarse con un concepto desconocido, investigarlo con apoyo de la LEC y sus compañeros también da cuenta de la flexibilidad didáctica que se presenta en esta aula.

En la preparación de la demostración pública, la alumna escribe en un rotafolio las siguientes preguntas: "Qué es la biodiversidad". "Qué es un ecosistema”. "Qué es genes". "Qué es una especie”. "Qué son las especies endémicas". Con ese cartel, Karime pasó al frente del grupo y, siguiendo "su guion", respondió una a una las preguntas. La demostración pública duró cerca de seis minutos.

Lo que Karime compartió en la demostración pública fue sobre lo que aprendió y no sobre cómo lo aprendió, aunque en su RPA sí hay manifestación de dificultades y expresiones de cómo procedió.

Al final se establece el siguiente diálogo:

\section{LEC: ¿Cómo se conforma la biodiversidad?}

K: $\quad$ La biodiversidad está conformada por los animales y muchas plantas.

LEC: $\quad$ Y a esos lugares, ¿cómo se les llama?

K: $\quad$ Ecosistemas.

LEC: ¿En México, con cuáles ecosistemas contamos?

K: $\quad$ El bosque, la selva, también el desierto, el desierto es un lugar donde no hay mucha fauna ni flora, porque es un lugar donde es muy caliente, de flora podemos encontrar cactus o nopales también, también víboras; en los bosques sí se pueden encontrar muchos animales porque hay mucha vegetación para que los animales coman... también hay mucha agua para que los animales puedan vivir.

De la demostración pública se puede mencionar que la información que Karime comparte con sus compañeros de aula es muy explicativa, llena de ejemplos, y usando un lenguaje que muestra apropiación de los conceptos y del tema en general. 


\section{CONSIDERACIONES FINALES}

Cabe advertir que no sabemos si este caso es representativo de las aulas en Conafe; sin embargo, sí podemos establecer que es un hecho que la relación tutora bien implementada es una alternativa para la educación de los niños, niñas y jóvenes de las regiones más apartadas de México. Una buena implementación permite que una LEC tenga un buen conocimiento de la función tutora, lo que permite que el alumnado escoja los temas, pues impulsa la investigación, apoya a los estudiantes para que encuentren su manera de resolver las dudas y desafíos, impulsa el trabajo autónomo, permite que el alumnado se dé tutoría y los y las estudiantes se apoyen para situaciones muy puntuales como la búsqueda de algún concepto en la computadora. Cabe resaltar que realiza todo lo anterior mientras presta atención adecuada y continua a todos y todas las estudiantes, pues vale recordar que es un aula muy diversa, típica de las multigrado, en edad, cultura, intereses y procesos, tal como lo indica Tomlinson (2008).

Un aspecto a resaltar es la condición del tiempo usado por la alumna para llevar a cabo su aprendizaje sobre biodiversidad pues, como lo menciona Vosniadou (2006, p. 27): “aprender es una tarea cognitiva compleja que no puede ser apresurada, requiere un tiempo considerable y periodos de práctica para comenzar a construir experiencia en el área”. De igual manera, los demás alumnos y alumnas se toman su tiempo para construir su aprendizaje, bien sea la lectoescritura, "Las figuras geométricas" o "La Revolución mexicana de 1910", temas todos sobre los que estaba trabajando el alumnado de esta aula.

El tiempo usado en esta experiencia trastoca la "gramática escolar" imperante en el sistema educativo, donde la enseñanza es del mismo modo, el ritmo de aprendizaje es impuesto igual para todos, es decir, el aprendizaje se supedita al tiempo de instrucción.

Por otro lado, con las descripciones de la relación tutora, el RPA y la demostración pública podemos afirmar que esta alumna tiene un "aprendizaje profundo", con base en la manera en que la alumna registra lo aprendido, tanto en la profundización con la información acerca de las especies endémicas, como en la extensión con los variados conceptos alrededor de la biodiversidad; de igual manera, 
se observa cómo la alumna muestra un grado de dominio importante; esto se evidencia con la forma en que escribe en su RPA y en la que habla en la demostración pública, usando ejemplos, mostrando evidencia, estableciendo analogías, y mostrando cómo usarlo en la vida cotidiana (por ejemplo, en "cómo harías para cuidarlas y protegerlas").

Al retomar los conceptos de dominio, identidad y creatividad planteados por Mehta y Fine (2019), se evidencia que:

- Respecto al dominio, la estudiante desarrolló conocimientos y habilidad, lo que fue expresado en el RPA y, posteriormente, en la demostración pública.

- En alusión a la identidad, se apreció el grado de interés y motivación que la estudiante manifestaba al estar trabajando, pues su atención estuvo conectada a lo que estaba aprendiendo.

- Con referencia a la creatividad, la estudiante -dentro del modelo ABCD- tiene previsto realizar una demostración pública, la que la interpelaba al desarrollo de la comunicación de lo aprendido ante sus compañeros y la LEC.

Al observar el proceso y el resultado del aprendizaje de la estudiante, se puede también decir que se atiene a las características planteadas por Valenzuela (2008), pues en el RPA se observa cómo la estudiante iba planteando las relaciones entre lo que sabía y lo que iba aprendiendo. Se aprecia cómo en este producto y en la demostración pública, la estudiante hace visible su profundización en el conocimiento disciplinar, logra determinadas extensiones del conocimiento con otras asignaturas (por ejemplo, cuando organiza información estadística en una gráfica). De esta forma, la estudiante demuestra dominio de lo aprendido cuando comparte explicaciones en la demostración pública o, en ocasiones, cuando la LEC la interpela, en esa misma demostración pública comunica lo que aprendió mediante ejemplos y hace ciertas analogías.

Por último, el presente texto es una contribución al campo del aprendizaje porque muestra claramente que es posible, mediante la relación tutora, disponer condiciones pedagógicas flexibles y portá- 
tiles para lograr aprendizajes profundos en contextos donde las condiciones sociales y económicas los dificultan, y donde las políticas públicas han sido históricamente inequitativas.

\section{REFERENCIAS}

Beas, J., Santa Cruz, J., Thomsen, P., y Utreras, S. (2001). Enseñar a pensar para aprender mejor. Santiago: Ediciones Pontificia Universidad Católica de Chile.

Biggs, J. (1993). What do inventories of students learning processes really measure? A theoretical review and clarification. British Journal of Educational Psychology, (63), 3-19. doi:10.1111/j.2044-8279.1993. tb01038.x

Cámara, G. (2013). Entramado conceptual. México: Redes de tutoría. http://redesdetutoria.org/entramado-conceptual/

Conafe (2016a). Marco curricular de la educación comunitaria. Modelo $A B C D$. Aprendizaje basado en la colaboración y el diálogo. México: Conafe. https://www.gob.mx/cms/uploads/attachment/file/411245/ Marco_Curricular.pdf

Conafe (2016b). Exploración y comprensión del mundo natural. México: Conafe. https://www.gob.mx/cms/uploads/attachment/file/139825/ UAA_ECMN_07-2016-4.pdf

Conafe (2016c). Unidades de aprendizaje autónomo 2016. México: Conafe. https://www.gob.mx/conafe/documentos/unidades-de-aprendizajeautonomo-2016

López Aguado, M., y López Alonso, A. (2013). Los enfoques de aprendizaje. Revisión conceptual y de investigación. Revista Colombiana de Educación, (64), 131-153. https://www.redalyc.org/ pdf/4136/413634076006.pdf

López, D. (2016). La relación tutora y la mejora del aprendizaje. Revista RED 1(3), 12-17. https://www.inee.edu.mx/wp-content/ uploads/2019/01/Red03-1PDF.pdf Páginas 12 -17

Mehta, J., y Fine, S. (2019). In Search of Deeper Learning: The Quest to Remake the American High School. Cambridge, Massachusetts: Harvard University Press. https://www.the74million.org/article/book-excerpton-the-hunt-for-americas-best-high-schools-a-harvard-professor-anda-teacher-find-disappointment-then-inspiration-hidden-in-plainsight/ 
Mejía, F., Pérez, I., y Frausto, A. (2016). Análisis de la propuesta de gestión pedagógica del nuevo modelo de educación comunitaria. México: ILCECEE.

Mejía, F., y Sierra, E. (2008). Metodología para el seguimiento cualitativo a escuelas con énfasis en situaciones áulicas (documento sin imprimir). México: CEE.

Rincón-Gallardo, S. (2012). Redes de tutoría académica. México: SEP.

Sigüenza, W., Arsuaga, A., García Alonso, O., y Martínez, E. (2015). Enfoques de aprendizaje antes y después del aprendizaje basado en problemas. Opción, 31(4), 927-945. https://www.redalyc.org/ pdf/310/31045569054.pdf

Soler, M., Cárdenas, F., Hernández, F., y Monroy, E. (2017). Enfoques de aprendizaje y enfoques de enseñanza: origen y evolución. Educación y Educadores, 20(1), 65-88.

Tomlinson, C. (2008). El aula diversificada: Dar respuestas a las necesidades de todos los estudiantes. Barcelona: Ediciones Octaedro.

Tyack, D., y Cuban, L. (2002). En busca de la utopia. Un siglo de reformas en las escuelas públicas. México: FCE.

Valenzuela, J. (25 de Julio de 2008). Habilidades de pensamiento y aprendizaje profundo. Revista Iberoamericana de Educación , 46(7), 2-9. https://rieoei.org/historico/deloslectores/2274Valenzuela.pdf

Vosniadou, S. (2006). Cómo aprenden los niños. Series prácticas educativas (7). México: Oficina Internacional de Educación, Academia Internacional de Educación, Centro Nacional de Evaluación para la Educación Superior, Consejo Mexicano de Investigación Educativa, Departamento de Investigaciones Educativas, Instituto Nacional para la Evaluación de la Educación, Universidad Pedagógica Nacional. https://www.uv.mx/rmipe/files/2017/02/Como-aprenden-los-ninos. pdf 